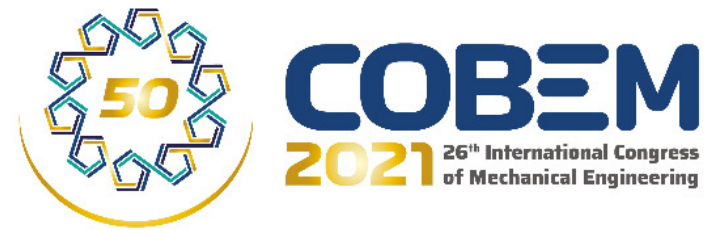

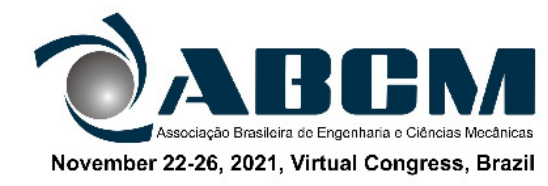

COBEM2021-1885

\section{REMARKS ON THE DYNAMIC BEHAVIOR OF AN ASYMMETRIC BISTABLE ENERGY HARVESTER}

\section{João Pedro Norenberg}

São Paulo State University - UNESP, Ilha Solteira, Brazil

jp.norenberg@unesp.br

\section{Roberto Luo}

Rio de Janeiro State University - UERJ, Rio de Janeiro, Brazil

cai.roberto@graduacao.uerj.br

\section{Americo Cunha Jr}

Rio de Janeiro State University - UERJ, Rio de Janeiro, Brazil

americo.cunha@uerj.br

\section{Samuel da Silva}

São Paulo State University - UNESP, Ilha Solteira, Brazil

samuel.silva13@unesp.br

\section{Paulo Sérgio Varoto}

University of São Paulo - USP, São Carlos, Brazil

varoto@sc.usp.br

Abstract. The current paper studies an asymmetric bistable energy harvesting system which presents a very complex dynamics due to the presence of geometric and material nonlinearities. The objective here is to understand how the system parameters can change the dynamic behavior and identify which parameters most affect the harvested power. For this purpose, we compute basins of attraction for different asymmetry degrees, which are controlled by the bias angle of the system. In addition, we perform the global sensitivity analysis based on the Sobol' indices to identify the individual and joint contributions of each parameter in the energy recovering process.

Keywords: Nonlinear Energy Harvesting, Basins of Attraction, Global Sensitivity Analysis, Sobol Indices

\section{INTRODUCTION}

Energy harvesting comprises recovering energy dispersed in the environment into a useful source, i.e., to generate electricity for low consumption and scale of electronic devices. It is a promising source due to the low growth rate of power supplies, like cells and batteries as appointed by Patel and Smith (2017). Energy harvesting has applications in medical implants, such as pacemaker that use the heartbeat vibration as energy sources (Karami and Inman, 2012); in the generation of bio-cellular energy through the potential difference existing in the plasma membrane of electrically polarized cells (Catacuzzeno et al., 2019); and self-power sensor networks for structural health monitoring using as source the automobile car vibration (Rana et al., 2020).

Among the possibilities of energy sources present in the environment (for instance, solar, wind, thermal, kinetic, radio and others), the kinetic energy from vibration motion has been explored for its wide application field. One advantages is that vibrations are available almost everywhere, on vehicles, aircraft, machines, human bodies, and civil buildings, for instance. It is worth mentioning that piezoelectric materials received rather attention in vibration application due to its simplicity, and high-power density.

However, vibrations diverge from each other for the frequency and amplitude, thus one challenge is to design a suitable energy harvester to couple with different sources of excitation. Linear energy harvesting devices have dynamic simplicity, but they usually provide reduced output power, limiting the operating frequency range of the harvesting system. To overcome this limitation, a broadband nonlinear vibration energy harvesters through bistable oscillators were proposed by Cottone et al. (2009) and Erturk et al. (2009), showed a substantial increase in the generation of piezoelectric energy concerning the linear system in a wide frequency band. Peterson et al. (2017); Lopes et al. (2017, 2019) analyzed a set of parameters which provided a chaotic or regular behavior in the dynamics. In engineering, chaos can be harmful and 
reduce the efficiency of a structure. In this context, de la Roca et al. (2019) explored the OGY method to control chaos on a bistable energy harvesting, showed that chaos can be useful to generate energy or spoil it, depending on the chaotic signal.

Although the bistable nonlinearities presented are beneficial in many conditions for energy harvesting, nonlinearities from asymmetries have proven to be harmful (Wang et al., 2018). Asymmetries correspond to a lack of symmetry, both geometric and dynamic, where inertial forces act asymmetrically. This characteristic is present in practically all real vibration energy harvesters, the reasons for which may be due to nonuniform manufacturing, the eccentricity of the buckling or magnetic force, as well as the influence of the inhomogeneity of the materials used. Therefore, considering these effects make a dynamic model more reliable and crucial for assessing the system performance.

In this way, understanding the physical phenomenon of asymmetric bistable energy harvester is necessary for elaborating a high-performance device. Due to the nonlinearities present, this task is complex and requires sophisticated tools to extract implicit system characteristics. Therefore, this work uses global sensitivity analysis and basins of attraction as an arsenal to tackle this task. Thus, here our objective is to evaluate the dynamic performance of the asymmetric bistable energy harvesting model and verify which parameters are more linked to this performance and can be further used in improving the harvesting capabilities of the system under investigation.

\section{ASYMMETRIC BISTABLE ENERGY HARVESTING MODEL}

The asymmetric bistable energy harvesting system consists of a cantilever ferromagnetic beam attached to a rigid base excited by a harmonic force, with two permanent magnets asymmetrically spaced from its free end causing asymmetric bistable nonlinearity, and a piezoelectric transducer coupled to the structure with an electrical circuit. The harvester also has a bias angle, which leads to oscillates over a certain angle $\phi$ and creates an external force from the self-weight of the harvester, as a dynamic asymmetry. An illustration of asymmetric bistable energy harvester can be seen in Figure 1

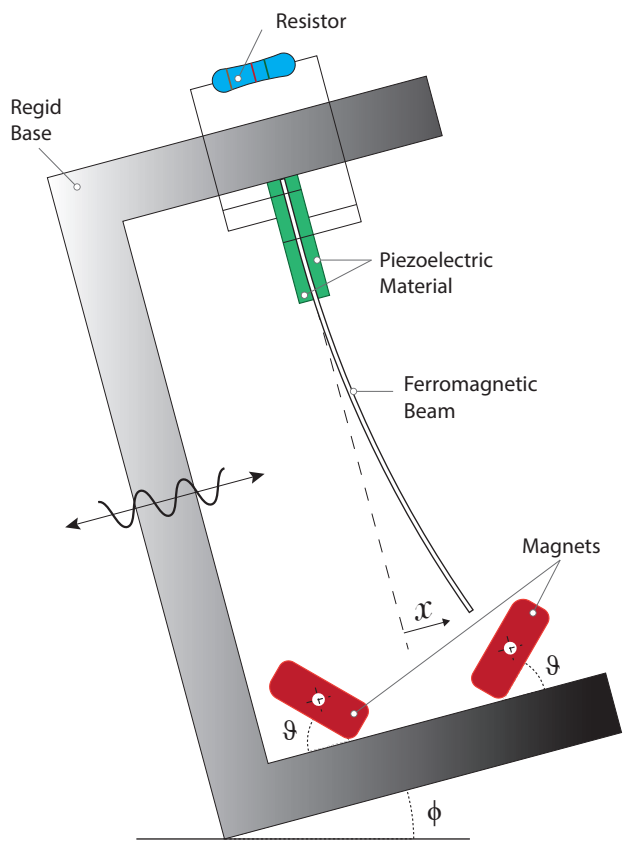

Figure 1: Schematic illustration of the asymmetric bistable energy harvester.

The dimensionless equations of motion for the asymmetric bistable energy harvester are

$$
\begin{aligned}
& \ddot{x}+2 \xi \dot{x}-\frac{1}{2} x\left(1+2 \delta x-x^{2}\right)-\chi v-p \sin \phi=f \cos (\Omega t), \\
& \dot{v}+\lambda v+\kappa \dot{x}=0, \\
& x(0)=x_{0}, \dot{x}(0)=\dot{x}_{0}, v(0)=v_{0},
\end{aligned}
$$

where $\xi$ is the damping ratio; $\chi$ is the piezoelectric coupling term in the mechanical domain; $\lambda$ is a reciprocal time constant; $\kappa$ is the piezoelectric coupling term in electrical domain; $\delta$ is asymmetric coefficient at energy potential; $f$ is the external excitation amplitude; $\Omega$ is the external excitation frequency. The initial conditions are $x_{0}, \dot{x}_{0}$ and $v_{0}$, which, respectively, represent the initial values of the beam edge position, velocity, and voltage over the resistor. 
The main quantity of interest (QoI) associated with the dynamical system is the mean output given by

$$
P=\frac{1}{T} \int_{t_{0}}^{t_{0}+T} \lambda v(t)^{2} d t
$$

which is the temporal average of the instantaneous power over a given time interval of length $T$.

\section{BASINS OF ATTRACTION}

The basins of attraction map the sensibility of a dynamical system with respect to the initials conditions, i.e., they associate initial conditions to a certain attractor reached by the dynamics in the steady state. First, the plane $x \times \dot{x}$ is discretized with an uniformly $600 \times 600$ numerical grid, then each point of this grid is used as initial condition in a numerical integration process that identifies the attractors, which are the limits-cycles in the phase space. Finally, they are catalogued in a color scheme, so that each color identify which initial condition leads to that attractor.

The 0-1 test for chaos Gottwald and Melbourne (2016) is used to numerically characterize the dynamics, determining if the system evolves to the chaotic or regular response. In this technique, the original state coordinates $(x(t), \dot{x}(t))$ are mapped into a pair of transformed coordinates define by the Euclidean extension

$$
\begin{aligned}
& p_{n}(c)=\sum_{j=1}^{n} x\left(t_{j}\right) \cos (j c), \\
& q_{n}(c)=\sum_{j=1}^{n} x\left(t_{j}\right) \sin (j c),
\end{aligned}
$$

which $c$ is a random value uniformly drawn in the support $[0,2 \pi)$ and $n=1,2, \ldots, N$. For regular behavior, the $p_{n}$ and $q_{n}$ coordinates show bounded motion; otherwise, for chaotic behavior, the $p_{n}$ and $q_{n}$ coordinates asymptotically behave as Brownian motion. Then, in order to analyze the diffusive (or non-diffusive), the mean square deviation is calculated by

$$
M_{n}(c)=\lim _{N \rightarrow \infty} \frac{1}{N} \sum_{j=1}^{N}\left(\left[p_{j+n}(c)-p_{j}(c)\right]^{2}+\left[q_{j+n}(c)-q_{j}(c)\right]^{2}\right),
$$

so that, the classifier is obtained by the correlation

$$
K_{c}=\lim _{N \rightarrow \infty} \frac{\operatorname{Cov}\left(t_{n}, M_{n}\right)}{\sqrt{\operatorname{Var}\left(t_{n}\right) \operatorname{Var}\left(M_{n}\right)}}
$$

where $M_{n}=\left(M_{1}, M_{2}, \ldots, M_{n}\right), t_{n}=\left(t_{1}, t_{2}, \ldots, t_{n}\right)$, Cov and $V a r$ are covariance and variance operators.

It is worthwhile to mention that, the $K_{c}$ is calculated for several c values and a median is computed, in order to a definitive value. Then, we can classify the dynamic behavior $K>0.8$ as chaotic behavior, $K<0.2$ as regular behavior and $K<0.8 \& K>0.2$ as an inconclusive behavior.

Figures 2 and 3 depict the basins of attractions and the attractors projections, respectively, in the initial displacement $\times$ initial velocity plane in a interval $\left(x_{0}, \dot{x}_{0}\right) \in[-2.5,2.5] \times[-2.5,2.5]$ for different bias angles. The following physical parameters are adopted: $f=0.091 ; \Omega=0.8 ; \xi=0.01 ; \chi=0.05 ; \lambda=0.05 ; \kappa=0.5$ and $\delta=0.15$.

Each color in the basins of attraction at Figure 2 represents a different solution that depends on the initial conditions and is presented in the phase spaces of Figure 3. When $\phi=-5^{\circ}$, the system has two solutions; the green basin refers to the green attractor (period-5 response); the blue basin represents a periodic-1 response of high energy. For $\phi=0^{\circ}, \phi=$ $5^{\circ}, \pm 15^{\circ}, \pm 25^{\circ}$ and $\phi=-35^{\circ}$ the system presents only two solutions, blue and red basins, which can be checked in the phase space. The red basins represent low energy orbits, which are undesirable for energy harvesting. When $\phi=35^{\circ}$ and $\phi=-40^{\circ}$, a yellow basin appears in addition to the blue and red basins, which has a high amplitude of oscillation around the right equilibrium point and low amplitude around the right equilibrium point. In addition, we can note that as the bias angle varies, the system can present larger and smaller limit cycles; for example, for small bias angles, the model is almost symmetrical. As the bias angle increases, there is an asymmetry in the basins. Note that the solution has a larger orbit limit cycle around the negative equilibrium point at negative angles. As the angle becomes positive, this condition is inverted, and limiting cycles of larger orbits around the positive equilibrium point are verified. In this way, asymmetry changes are due to bias angle changes. It is worth illustrating that for negative angles, the basins show greater asymmetries than for positive angles. Also, the asymmetry of the quadratic coefficient $(\delta)$ can be compensated through the bias angle. To illustrate it, we can verify that $\phi=15^{\circ}$ has a smaller asymmetry than $\phi=-15^{\circ}$. Also, when $\phi=0^{\circ}$, the asymmetry existing in the system is only coming from the coefficient $\delta$. For $\phi=-5^{\circ}$, we note a particular behavior, such that the dynamics present only high energy attractors (periods 1 and 5). It can be explained by an inversion asymmetry region. 


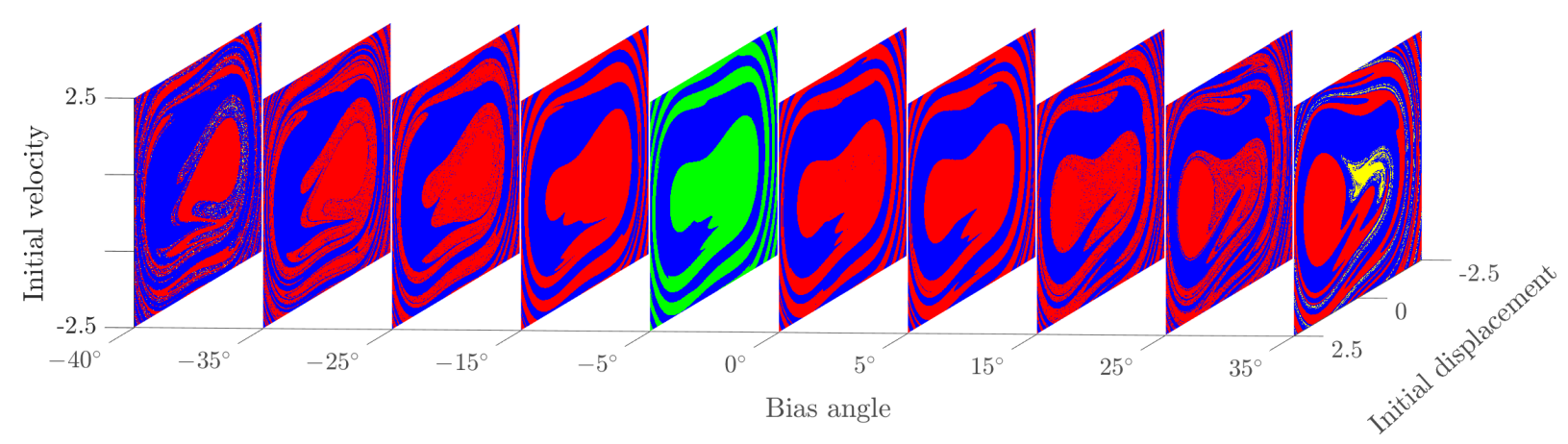

Figure 2: Basins of attraction in initial displacement versus initial velocity plane $\left(x_{0}, \dot{x}_{0}\right) \in[-2.5,2.5] \times[-2.5,2.5]$ for different bias angle $\phi \in\left\{-40^{\circ},-35^{\circ},-25^{\circ},-15^{\circ},-5^{\circ}, 0^{\circ}, 5^{\circ}, 15^{\circ}, 25^{\circ}, 35^{\circ}\right\}$.

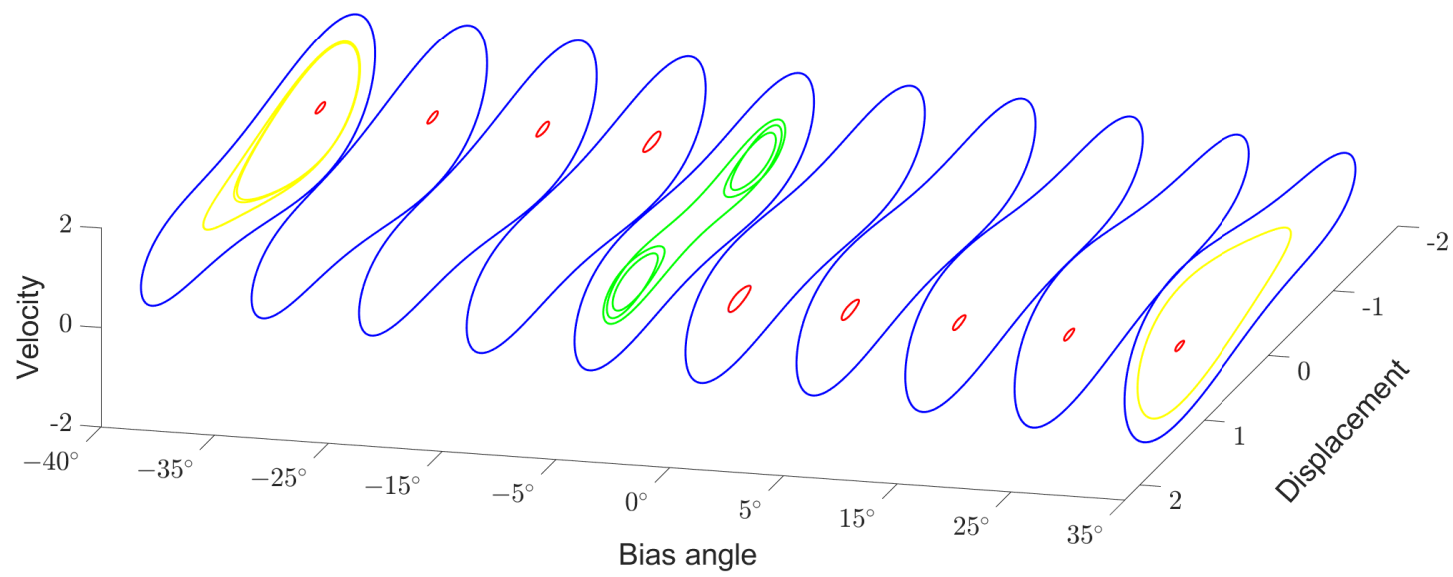

Figure 3: Attractors corresponding to the colors in the basins of attraction.

Based on these analyses, we can conclude that this asymmetric energy harvester has a very complex dynamics behavior. Besides the initial conditions and inclination angles, excitation conditions and physical parameters can be varied. Thus, evaluate potential solutions to enhance the energy recovery, such as done by Cunha Jr (2021), is a non-trivial task. Therefore, to analyze the influence of all these parameters, a global sensitivity analysis study is employed.

\section{SOBOL SENSITIVITY ANALYSIS}

Global Sensitivity Analysis (GSA) is a statistical tool that has been receiving great attention in the analysis of many engineering systems, providing a comprehensive approach on how parametric variation can affect the underlying system response. The key point here is to evaluate the degree of influence of the parameters, and their joint effect, on the system response. Sobol' indices is a GSA technique that employs an orthogonal decomposition in terms of conditional variances, making it possible to measure the (individual and/or joint) effects of the system parameters variability on the average power (quantity of interest). In the nonlinear dynamical systems analysis, is can be very useful tool to reveal the influence of each nonlinearity, especially because nonlinear systems, in general, present large sensitivity to variations on the input parameters and initial conditions, as shown previously.

The dynamical system of interest can be abstractly represented by

$$
Y=\mathcal{M}(\mathbf{X}), \quad \mathbf{X}=\left\{X_{1}, X_{2}, \ldots, X_{k}\right\}
$$

where $\mathbf{X}$ is an input vector with $k$ independent parameters, that is modified by the mathematical operator $\mathcal{M}$, to produce the scalar output $Y$ (the QoI). 
Equation 9 can be written as decomposition into summands of different dimensions defined by Sobol (1993), a.k.a, Hoeffding-Sobol decomposition (Sudret, 2008):

$$
Y=\mathcal{M}_{\mathrm{o}}+\sum_{i=1}^{k} \mathcal{M}_{i}\left(X_{i}\right)+\sum_{i<j} \mathcal{M}_{i j}\left(X_{i}, X_{j}\right)+\ldots+\mathcal{M}_{1 \ldots k}\left(X_{1} \ldots X_{k}\right),
$$

which all the sums of the expansion can be recursively computed, with the first term $\mathcal{M}_{\mathrm{o}}$ a constant equal to the expected value of $Y, \mathcal{M}_{i}\left(X_{i}\right)$ and $\mathcal{M}_{i j}\left(X_{i}, X_{j}\right)$ terms are the conditional expected value for parameter $i$ and $i j(i \neq j)$, respectively and so on. As this decomposition is orthogonal, it can be rewritten as a variance decomposition. After all, for independent input variables, we can quantify the contribution of the individual $X_{i}$ and combination $X_{i j}, \ldots, X_{i \ldots k}$ of variables to the total variance $\operatorname{Var}[Y]$, which is called as Sobol indices. The first-order Sobol indices $S_{i}$ quantify the additive effect of each input separately concerning the total variance

$$
S_{i}=\frac{\operatorname{Var}\left[\mathcal{M}_{i}\left(X_{i}\right)\right]}{\operatorname{Var}[\mathcal{M}(\mathbf{X})]}
$$

and the interaction effects of the inputs, as second-order Sobol indices $S_{i j}$

$$
S_{i j}=\frac{\operatorname{Var}\left[\mathcal{M}_{i j}\left(X_{i}, X_{j}\right)\right]}{\operatorname{Var}[\mathcal{M}(\mathbf{X})]} .
$$

Table 1 presents the range values for each parameter of the system that we use for the Sobol indices calculation. The distribution function used is uniform. The variabilities are employed concerning the nominal values previously adopted. The $\phi$ parameter is used as a control parameter with different values.

Table 1: Range of values used for the parameters of asymmetric bistable energy harvester.

\begin{tabular}{l|c|c}
\hline Feature & Parameter & Range \\
\hline Damping ratio & $\xi$ & $\pm 20 \%$ \\
Piezoelectric coupling mechanical domain & $\chi$ & $\pm 20 \%$ \\
Reciprocal time constant & $\lambda$ & $\pm 20 \%$ \\
Piezoelectric coupling electrical domain & $\kappa$ & $\pm 20 \%$ \\
Amplitude of excitation & $f$ & $\pm 20 \%$ \\
Frequency of excitation & $\Omega$ & $\pm 20 \%$ \\
Asymmetric potential coefficient & $\delta$ & {$[-0.150 .15]$} \\
Bias angle & $\phi$ & control \\
Initial displacement & $x_{0}$ & {$[-2.52$} \\
Initial velocity & $\dot{x}_{0}$ & {$\left[\begin{array}{ll}-2.5 & 2.5\end{array}\right]$} \\
\hline
\end{tabular}

Toolbox elaborated by Marelli and Sudret (2014) is used to compute Sobol indices. Figure 4 presents the first-order Sobol indices and the second-order indices (only larger indices) for $\phi$ equal to $\pm 5, \pm 15, \pm 25$ and \pm 35 . The quantity of interest evaluated is the mean power (Eq. 4).

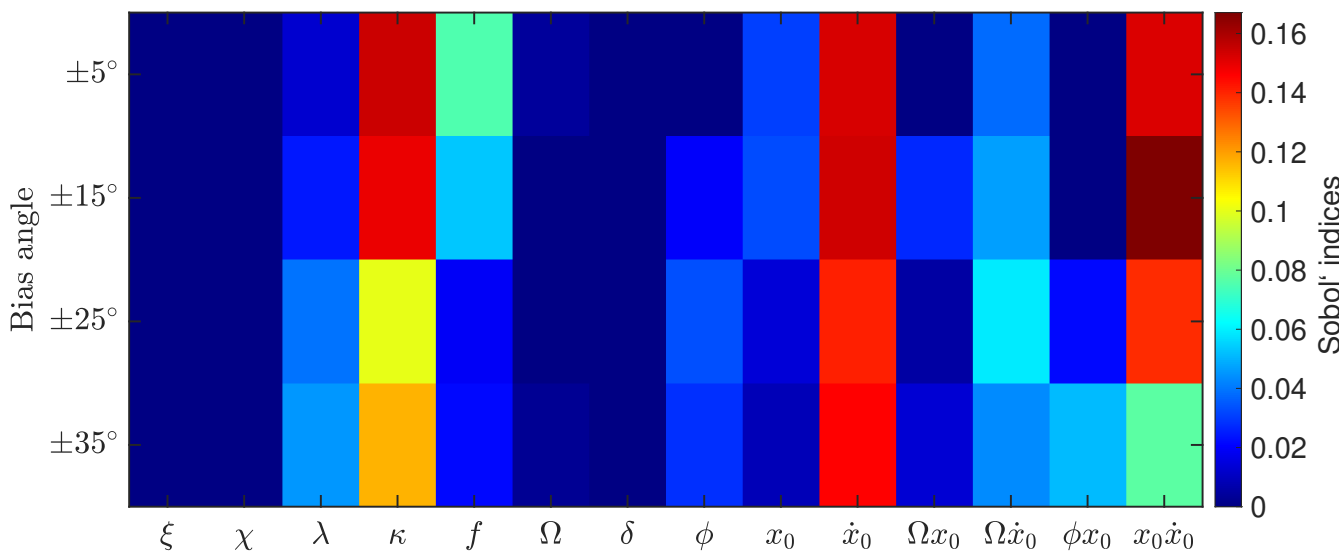

Figure 4: First- and second-order Sobol' indices based on mean power harvested under different bias angle ranges. 
As higher the Sobol index more sensitive these parameters on the power harvested. Initial velocity is the most influential first-order parameter for all $\phi$ 's. Variations in the initial velocity generate changes in the mean power harvested. Next is $\kappa$, piezoelectric coupling in the electrical domain, and that for smaller bias angle the effect is amplified. $f, \phi, \lambda$ and $x_{0}$ have a lower sensitivity. Finally, individual effects of $\Omega, \xi, \chi$ and $\delta$ are negligible.

The second-order indices that quantify the joint effect, the initial conditions $\left(x_{0} \dot{x}_{0}\right)$ are highlighted, in which they presented significant values. For $\phi= \pm 15^{\circ}, x_{0} \dot{x}_{0}$ has greater effect than first-order. For $\phi= \pm 35^{\circ}, x_{0} \dot{x}_{0}$ shows an influence decrease, since $x_{0}$ and $\dot{x}_{0}$ also show decreases in their respective individual influences. In addition, the effects combined with the parameter $\Omega$ stand out, which for first-order indices has negligible sensitivity. Higher-order indices there are minor influences.

Overall, the Sobol' indices are efficient to demonstrate which parameters most influence the mean power harvested. Likewise seen in the basins of attraction, the initial velocity alters the system response, which dominates the variability of other parameters. Despite $\phi$ controls the asymmetry, we showed it is not predominant in the mean power sensitivity. Alterations in the basins, presented by different values of $\phi$, do not affect energy generation, and we can illustrate this in the orbits at similar phase spaces. Thus, the mean power variance is small when varying $\phi$. Besides, $\kappa$ stands out as it increases or decreases, the mean power changes, since it is an electromechanical coupling term, so it is directly linked to the recovery of electricity.

Therefore, we can conclude that the sensitivity analysis provides valuable knowledge for dynamic study. Also, it is a crucial tool to examine the influences of parameters with higher indices on the mean power harvested. Thus, it reduces the design's complexity and provides non-trivial insights that could not be identified in the basins of attraction, demonstrating a helpful tool to complement the system knowledge.

\section{FINAL REMARKS}

This work has analyzed the nonlinear dynamics of an asymmetric bistable energy harvesting device employing basins of attraction and global sensitivity analysis. The dynamics behavior was addressed to characterize the sensibility of the system response to the initial conditions and the influence of bias angle. Basins of attraction were computed for the different bias angles that lead to asymmetry degree changes. Then, we identified initial conditions sets of values that provide different behaviors and showed dynamics complexity. Global sensitivity analysis based on Sobol indices was performed under a distinct bias angle. The results obtained show which parameters most affect the recovered mean power, therefore reinforcing, as already mentioned in the previous section that GSA through Sobol Indices is, in fact, a very useful tool to be employed in the design and optimization of bistable energy harvesting systems.

\section{ACKNOWLEDGEMENTS}

The authors are indebted to the Brazilian institutions CNPq, CAPES, FAPESP, FAPERJ and ABCM for the financial support given to this research. The authors also thank Prof. Marcelo Savi (UFRJ), for useful discussions concerning the problem addressed in this work.

\section{REFERENCES}

Catacuzzeno, L., Orfei, F., Di Michele, A., Sforna, L., Franciolini, F. and Gammaitoni, L., 2019. “Energy harvesting from a bio cell". Nano Energy, Vol. 56, p. 823.

Cottone, F., Vocca, H. and Gammaitoni, L., 2009. “Nonlinear energy harvesting”. Physical Review Letters, Vol. 102, p. 080601.

Cunha Jr, A., 2021. "Enhancing the performance of a bistable energy harvesting device via the cross-entropy method". Nonlinear Dynamics, Vol. 103, pp. 137-155. doi:10.1007/s11071-020-06109-0.

de la Roca, L., Peterson, J.V.L.L., P, M. and Cunha Jr, A., 2019. "Control of chaos via ogy method on a bistable energy harvester". In Proceedings of the 25th International Congress of Mechanical Engineering - COBEM 2019. Uberlândia, Brazil.

Erturk, A., Hoffmann, J. and Inman, D., 2009. “A piezomagnetoelastic structure for broadband vibration energy harvesting”. Applied Physics Letters, Vol. 94, p. 254102.

Gottwald, G. and Melbourne, I., 2016. "The 0-1 test for chaos: A review". Chaos Detection and Predictability, Vol. 915, pp. 221-247.

Karami, M.A. and Inman, D.J., 2012. "Powering pacemakers from heartbeat vibrations using linear and nonlinear energy harvesters". Applied Physics Letters, Vol. 100, p. 042901.

Lopes, V.G., Peterson, J.V.L.L. and Cunha Jr, A., 2017. "Numerical study of parameters influence over the dynamics of a piezo-magneto-elastic energy harvesting device”. In XXXVII Congresso Nacional de Matemática Aplicada e Computacional (CNMAC 2017). São José dos Campos, Brazil.

Lopes, V.G., Peterson, J.V.L.L. and Cunha Jr, A., 2019. "Nonlinear Characterization of a Bistable Energy Harvester 
Dynamical System". In M. Belhaq, ed., Topics in Nonlinear Mechanics and Physics: Selected Papers from CSNDD 2018 (Springer Proceedings in Physics), Springer, Singapore, pp. 71-88.

Marelli, S. and Sudret, B., 2014. "UQLab: a framework for uncertainty quantification in MATLAB". In Proc. 2nd Int. Conf. on Vulnerability, Risk Analysis and Management (ICVRAM2014), Liverpool, United Kingdom. p. 2554-2563. doi:10.1061/9780784413609.257.

Patel, S.N. and Smith, J.R., 2017. "Powering pervasive computing systems". Pervasive Computing Revisited, Vol. 16, pp. 32-38.

Peterson, J.V.L.L., Lopes, V.G. and Cunha Jr, A., 2017. "On the nonlinear dynamics of a bi-stable piezoelectric energy harvesting device". In Proceedings of the 24th International Congress of Mechanical Engineering - COBEM 2017. Curitiba, Brazil.

Rana, M.D., Khan, A.A., Huang, G., Mei, N., Saritas, R., Wen, B., Zhang, S., Voss, P., Abdel-Rahman, E., Leonenko, Z., Islam, S. and Ban, D., 2020. "Porosity modulated high-performance piezoelectric nanogenerator based on organic/inorganic nanomaterials for self-powered structural health monitoring". Applied Materials \& Interfaces, Vol. 12, pp. 47503-47512.

Sobol, I., 1993. "Sensitivity estimates for nonlinear mathematical models". Mathematical and Computer Modelling, Vol. 1, p. 407.

Sudret, B., 2008. "Global sensitivity analysis using polynomial chaos expansions". Reliability Engineering and System Safety, Vol. 93, No. 7, pp. 964-979.

Wang, W., Cao, J., Bowen, C. and Litak, G., 2018. "Multiple solutions of asymmetric potential bistable energy harvesters: numerical simulation and experimental validation”. The European Physical Journal B, Vol. 91.

\section{RESPONSIBILITY NOTICE}

The authors are the only responsible for the printed material included in this paper 